\title{
Puntos de encuentro entre el enfoque pedagógico de Paulo Freire y la Educación en Derechos Humanos
}

\author{
Meeting points between Paulo Freire pedagogical focus \\ and Human Rights Education
}

\author{
Allyson Núñez Méndez ${ }^{1}$ \\ Universidad Estatal a Distancia, Costa Rica
}

\begin{abstract}
Resumen. El objetivo principal de la investigación fue comprender la forma en la que son afectad Resumen. La propuesta pedagógica de Paulo Freire genera los elementos tanto para una educación dirigida al cambio social como hacia el enfoque de la Educación en Derechos Humanos. Este ensayo se propone como objetivos, primero, profundizar en los elementos que incluye el enfoque de Educación en Derechos Humanos; segundo, exponer los principales conceptos de la pedagogía de Paulo Freire y, tercero, demostrar que el enfoque de la Educación en Derechos Humanos es una de las formas más concretas de vinculación entre las tendencias en educación y la pedagogía de Paulo Freire. La Educación en Derechos Humanos se dirige a hacer de la persona un sujeto de derecho, con una gran capacidad de cuestionamiento crítico de la sociedad, que logra convertirse en un agente de cambio social, comprometido en el cumplimiento de los derechos humanos. Para lograr este camino, es necesario un docente que eduque para la crítica y para el cambio, por medio del diálogo. La metodología empleada para la elaboración de este documento consistió en una revisión y un análisis profundo de los textos sobre el tema. Se concluyó que ambos enfoques educativos tienen coincidencias fundamentales sobre la esencia de la educación y el hecho educativo.
\end{abstract}

Palabras claves: Paulo Freire, Pedagogía de la esperanza, Educación en Derechos Humanos, sujeto de derecho, diálogo educativo.

Abstract: Paulo Freire's pedagogical approach generates the elements both for an education for the social change and towards the approach of Human Rights Education. This essay proposes as objectives, first, to delve into the elements included in the Human Rights Education approach; second, to expose the main concepts of Paulo Freire's pedagogy and, third, to demonstrate that the approach to Human Rights Education is one of the most concrete ways of linking between trends in education and Paulo Freire's pedagogy. Human Rights Education is aimed at making the person a subject of law, with a great capacity for critical questioning society, which manages to become an agent of social change, committed to the fulfillment of human rights. To achieve this path, you need a teacher who educates for criticism and for change, through dialogue. The methodology used for the preparation of this document consisted of a review and in-depth analysis of the texts on the subject. It was concluded that both educational approaches have fundamental coincidences on the essence of education and the educational fact.

Keywords: Paulo Freire, pedagogy of hope, Education in Human Rights, subject of law, educational dialogue.

\footnotetext{
${ }^{1}$ Máster en Derechos Humanos, candidata a doctorado en Educación, Universidad Estatal a Distancia, Costa Rica. Dirección electrónica: anunez@uned.ac.cr
} 


\section{Introducción}

Educar con perspectiva de derechos humanos implica una necesidad en la sociedad actual. Requiere una posición pedagógica que acerque la normativa jurídica y política a la práctica docente favorecedora del aprendizaje y la formación de las personas para ser sujetos de derechos. Este enfoque ayuda a ver el mundo desde la dignidad de la persona humana y como guía para crear una nueva cultura donde los derechos humanos se consideren como ejes reguladores de las relaciones entre los individuos.

Existen muchas formas de acercarse a esta práctica educativa, entre ellas se destaca el pensamiento de Paulo Freire, expuesto en La pedagogía de la esperanza. Según esta investigadora, ofrece una manera acertada para entender y aproximarse a la praxis educativa en derechos humanos, su defensa y promoción, en correspondencia con su concepción de la educación como una práctica liberadora, y eje para la transformación de la sociedad (Freire, 2015).

La Educación en Derechos Humanos debe ser vista como un verdadero aprendizaje de la realidad social; en razón de ello, debe referir a todo aquel aspecto que influye en la visión de mundo, así como en el modo de vida de los seres humanos, junto con las costumbres y los valores del sujeto: todos los anteriores, aspectos acordes con el pensamiento de Freire, quien toma en cuenta la realidad de las personas como un factor determinante para realizar la práctica educativa, porque "no hay ni ha habido jamás una práctica educativa, en ningún espacio-tiempo, comprometida únicamente con ideas preponderantemente abstractas e intocables" (Freire, 2005, p. 101).

El presente trabajo se propone, primero, profundizar en los elementos que incluye el enfoque de Educación en Derechos Humanos; segundo, exponer los principales conceptos de la pedagogía de Paulo Freire y, tercero, demostrar que el enfoque de la Educación en Derechos Humanos es una de las formas más concretas de vinculación entre las tendencias en educación y la pedagogía de Paulo Freire.

Para la elaboración de este ensayo de tipo argumentativo, se consideró pertinente el uso de la investigación documental, la cual, por sus características, además de ser la búsqueda, recolección y procesamiento de información, se asume que también es "la presentación sistemática, coherente $\mathrm{y}$ suficientemente argumentativa de nueva información en un documento científico" (Tancara, 1993, p. 94), lo que posibilitó el reconocimiento de elementos que explican la pertinencia de los postulados de la pedagogía de la esperanza, propuesta por Paulo Freire, en la praxis de la Educación en Derechos Humanos.

La ruta que se siguió para esta investigación constó de tres momentos diferentes. En primer lugar, se buscó y recopiló textos relacionados con la línea de investigación en Educación en Derechos Humanos. En segundo lugar, se escogió la bibliografía necesaria para la definición de los conceptos básicos para este estudio: pedagogía de la esperanza, diálogo educativo, y sujeto de derecho. Esto permitió seleccionar con precisión las fuentes para explicar la función de la educación como una práctica transformadora de la sociedad.

Para cerrar en un tercer momento, se analizaron los documentos de Paulo Freire y se escogieron principalmente cuatro libros: La pedagogía del oprimido (1970), La pedagogía de la autonomía (1996), La pedagogía de la esperanza (2005) y La educación como práctica de la libertad (2015).

A partir de estos fundamentos teóricos, se dilucidaron, relacionaron $\mathrm{y}$ 
sintetizaron las ideas que cimentaron las conclusiones expuestas en el ensayo.

\section{El concepto de educación}

En este estudio, partimos de que la educación debe estar centrada en el estudiante para lograr el desarrollo de habilidades, conocimientos y hábitos para el ejercicio del pensamiento crítico, de manera que sea capaz de cuestionar la sociedad en la que vive, el poder, las desigualdades y el cambio social (Magendzo, 2007).

De esta manera, el desafío durante el proceso educativo consiste en desarrollar un pensamiento de complejidad, que, por una parte, incluya saberes que estaban dispersos; por otra parte, que promueva un cambio de actitud para organizar estos con el propósito de favorecer patrones individuales de empleo del intelecto, de pensamiento propio ante circunstancias específicas, con cabida para la curiosidad y la creatividad; a la vez, también para estimular la autonomía cognitiva, la capacidad de dudar, la actitud para problematizar y cuestionarse sobre sí mismo (a).

Igualmente, se requiere que la persona estudiante logre fortalecer habilidades y actitudes comunicativas que posibiliten el desarrollo de un pensamiento autónomo "estructurado reflexivamente y con disposición a la crítica y al diálogo; la disposición a aceptar y a respetar los puntos de vista divergentes apreciando el aporte de estas actitudes para la formación personal y la convivencia democrática" (Magendzo, 2007, p.11) con miras a que los seres humanos tomemos una conciencia crítica que nos lleve a obtener un mayor control sobre nuestras vidas y a la creación de una sociedad más justa.

Lo que además ayudará a preparar para la vida, a aprender a conocer el mundo, a "ver la totalidad, la multiplicidad de acciones posibles, desarrollar visiones de largo plazo, y favorecer el disfrute pleno de la vida en todas sus manifestaciones" (Morin, 2008, p. 4).

Este tipo de persona inspira la necesidad de que la educación se convierte en un instrumento para que las personas estudiantes como colectivo se capaciten para participar en la discusión valiente de su problemática, que los advierta de los peligros de su tiempo para que, consciente de ellos, ganen la fuerza y el valor para luchar, en lugar de ser arrastrados a la perdición de su propio "yo", sometido a las prescripciones ajenas (Freire, 2015, p. 84).

Todo lo anterior surge a partir de un proceso constructivo de diálogo entre los distintos protagonistas activos del desarrollo educativo, que contemple la formación integral de la persona, que involucra valores como el respeto y la tolerancia, así como también la búsqueda de la paz, la igualdad y la no violencia. Este diálogo predispondrá a las personas "a constantes revisiones, a análisis críticos de sus 'descubrimientos', a una cierta rebeldía, en el sentido más humano de la expresión, que lo identifique, en fin, con métodos y procesos científicos" (Freire, 2015, p. 84).

Se precisa de individuos que sean capaces de utilizar el conocimiento adquirido y potenciarlo permanentemente para poder adaptarlo a las nuevas situaciones que se presentan al seleccionar lo que aprenden. Sería de esperarse que este aprendizaje se produzca a lo largo de toda la vida, lo cual se manifestará en la autonomía de la persona, su juicio crítico y el desarrollo de todos sus talentos y aquellas capacidades que fortalezcan su responsabilidad personal para lograr el destino colectivo.

Precisamente, la Organización de las Naciones Unidas para la Educación, la Ciencia y la Cultura (Unesco) indica que la educación debe estar "orientada a 
explotar los talentos y capacidades de cada persona y a desarrollar la personalidad del educando, con el objeto de que mejore su vida y transforme la sociedad" (Unesco, 2000 , p. 3). Esta visión de la educación como transformadora de vida, exige el desarrollo de condiciones necesarias para que funcionen las instituciones y los programas de aprendizaje en cantidad suficiente para el acceso a ella de manera sencilla y factible, que se tomen en cuenta puntos de vista como la no discriminación, la accesibilidad física y la accesibilidad económica.

También, se requiere que los programas de estudio y los métodos pedagógicos sean aceptables, pertinentes, culturalmente adecuados y de buena calidad para todas las personas y que el sistema educativo tenga la suficiente flexibilidad para adaptarse a las necesidades de la sociedad y de las comunidades, de manera tal que las partes respondan a los requerimientos de las personas en contextos culturales y sociales variados.

En congruencia con un tipo de educación con estas características, es necesario implementar una práctica pedagógica, que, desde una posición controversial, plantee...

... temas, contenidos, objetivos y situaciones, problemáticas [problemáticos] y controversiales referidos, tanto a los problemas que la sociedad enfrenta en el presente en el plano político, económico, social y cultural en que los derechos humanos están involucrados, como [a] las controversias de derechos [sic] que se presentan en la vida cotidiana de los estudiantes, de sus familias y comunidades. (Magendzo, 2015, p.51).

Vista así, la educación promoverá el respeto y la dignidad de la persona.
Asimismo, colaborará con la formación de un ser humano que se empodere al reconocer su capacidad para realizar una transformación de la sociedad.

\section{La Educación en Derechos Humanos}

Según afirma Magendzo (2004), la Educación en Derechos Humanos "en su concepción de modernidad nació para formar ciudadanos" (p.7) y "adquiere sentido en la medida que entrega al educando un modo de ubicarse en la sociedad" (p.25). Este tipo de educación se orienta a que la persona tenga conocimiento de su situación dentro del cuerpo social, lo cual significa que pueda referirse al concepto de los derechos humanos, como sujeto de ellos y a las instituciones que los protegen $\mathrm{y}$ a las cuales puede recurrir cuando necesite su defensa.

También, se destina a que sea conocedora de la importancia de la promoción y defensa de las igualdades en lo que a sí mismo y a los demás afecta. Está familiarizada "con la Declaración Universal de Derechos Humanos y con algunas resoluciones, pactos, convenciones y declaraciones nacionales e internacionales, relacionadas [-os] con los derechos humanos" (Magendzo, 2003, p. 23). La formación en el tema de los derechos humanos es la base para el empoderamiento de la persona como sujeto de derechos.

El enfoque de Educación en Derechos Humanos parte de la misma Declaración Universal de Derechos Humanos, que, en su artículo 26.2, establece el hecho de que, las personas tengan un determinado nivel de instrucción, es necesario que reciban una educación específica para lograr alcanzar el pleno desarrollo de la personalidad y asegurarles el respeto por sus derechos y libertades fundamentales. Igualmente, hay un nutrido cuerpo normativo que le da 
solidez teórica a la Educación en Derechos Humanos.

De forma general, se le considera un derecho vinculado con los ámbitos económico, social y cultural. Específicamente, el artículo N. ${ }^{\circ} 13$ del Pacto Internacional de Derechos Económicos, Sociales y Culturales (ONU, 1966) establece que los Estados partes deben garantizar el derecho de toda persona a la educación. Asimismo, es necesario para garantizar y consagrar otros derechos.

Así lo establece en "Observación general N. ${ }^{\circ} 13$ ", del Comité de Derechos Económicos, Sociales y Culturales de Naciones Unidas (1999), en la oración inicial del párrafo 1 donde específica que "la educación es un derecho humano intrínseco y un medio indispensable de realizar otros derechos humanos". De igual forma, este mismo Comité explica en el párrafo 5 que el artículo 13 del Pacto debe ser interpretado en conjunto con otros diversos instrumentos que contribuyen a enriquecer los atributos que integran el derecho a la educación y que los Estados partes se han comprometido a respetar y concretar.

En el párrafo 43 de la "Observación general N. ${ }^{\circ} 13$ ", el Comité ha precisado que los Estados tiene la obligación de garantizar el derecho para poder ejercer los demás derechos sin discriminación alguna y así lograr la plena aplicación del artículo N. ${ }^{\circ} 13$ del Pacto. A su vez, en el párrafo 44 , se indica que el ejercicio del derecho a la educación se debe dar gradualmente a lo largo del tiempo, y las obligaciones que los Estados partes tienen son permanentes y concretas, es decir, la obligación por parte del Estado de aplicar el artículo N. ${ }^{\circ} 13$ no se pierde en ningún momento a pesar del paso del tiempo.

En la Convención de Derechos del Niño de 1989, específicamente el artículo
28, contiene un corolario que menciona que los Estados reconocen el derecho del niño a la educación y que esta debe fomentar su desarrollo en sus distintas formas. En el artículo 29 se establece que los Estados acuerdan que la educación debe velar por el desarrollo de la personalidad, las aptitudes y la capacidad mental y física del niño. En el inciso b del mismo artículo se establece que la educación debe infundir en el niño el respeto por los derechos humanos, las libertades fundamentales y los principios consagrados en la Carta de las Naciones Unidas.

En estos artículos se define y enmarca la educación como aquella que provee el desarrollo integral en los aspectos biológico, cognitivo, socioafectivo, por medio de experiencias de socialización pedagógica y recreativa.

Haciendo referencia a la educación con un propósito más amplio y abierto, dirigida a personas de cualquier edad y condición, en la "Conferencia Mundial sobre Educación para Todos" celebrada en Jomtien (Tailandia), en 1990, se estableció que existen necesidades básicas de aprendizaje que se deben definir y que cada persona debe estar en condiciones de aprovechar las oportunidades educativas ofrecidas para satisfacer sus necesidades de aprendizaje.

Para ello, se utilizan herramientas esenciales tales como la escritura, la lectura y los contenidos básicos de aprendizaje, los cuales son indispensables "para que los seres humanos puedan sobrevivir, desarrollar plenamente sus capacidades, vivir y trabajar con dignidad, participar plenamente en el desarrollo, mejorar la calidad de su vida, tomar decisiones fundamentadas y continuar aprendiendo" (Unesco, 1990, p. 1).

No es cualquier educación la que contribuye a que las personas vivan plenamente su dignidad de ser humano, en libertad y en paz. Debe ser una que 
busque preservar estos valores fundamentales, así como la comprensión, la tolerancia y amistad entre las naciones y los grupos. Entonces, de esta manera, promoverá el mantenimiento de la armonía entre las sociedades.

La Asamblea General de las Naciones Unidas (2006) define la Educación en Derechos Humanos como un conjunto de actividades de capacitación y difusión de información orientadas a crear una cultura universal en la esfera de los derechos humanos mediante la transmisión de conocimientos, la enseñanza de técnicas y la formación de actitudes, con la finalidad de:

a) Fortalecer el respeto de los derechos humanos y las libertades fundamentales;

b) Desarrollar plenamente la personalidad humana y el sentido de la dignidad del ser humano;

c) Promover la comprensión, la tolerancia, la igualdad entre los sexos y la amistad entre todas las naciones, los pueblos indígenas y los grupos raciales, nacionales, étnicos, religiosos y lingüísticos;

d) Facilitar la participación efectiva de todas las personas en una sociedad libre y democrática en la que impere el Estado de derecho;

e) Fomentar y mantener la paz;

f) Promover un desarrollo sostenible centrado en las personas y la justicia social. (pp. 4-5).

El Instituto Interamericano de Derechos Humanos (2002) explica este tipo de educación como un proceso mediante el cual las personas van conociendo y comprendiendo los propios derechos y los de los demás para reivindicarlos, de conformidad con la normativa establecida en los diferentes instrumentos internacionales, así como en la jurisprudencia interna. Esto quiere decir lo siguiente:

que todas las personas independientemente de su sexo, origen nacional o étnico y sus condiciones económicas, sociales o culturales- tienen la posibilidad real de recibir educación sistemática, amplia y de buena calidad que les permita: comprender sus derechos humanos y sus respectivas responsabilidades; respetar $\mathrm{y}$ proteger los derechos humanos de otras personas; entender la interrelación entre derechos humanos, estado de derecho y gobierno democrático; y ejercitar en su interacción diaria valores, actitudes y conductas consecuentes con los derechos humanos y los principios democráticos. (IIDH, 2002, p.15).

Este concepto de educación es reiterado en los documentos que emanan del Foro Mundial sobre la Educación, realizado en Incheon, República de Corea, en el 2015, porque amplía su ámbito al incluir elementos para una educación inclusiva y equitativa de calidad, que promueva oportunidades de aprendizaje permanentes para todas las personas. De igual forma, presenta una visión de la educación como transformadora de vidas y reconoce el importante rol que tiene como un factor principal para el desarrollo.

Dicho Foro (2015) rescata el carácter humanístico de la educación y su relación con el desarrollo, enlazándola con los derechos humanos, la dignidad, la justicia social, la inclusión, la protección, la diversidad cultural lingüística y la responsabilidad, además de la rendición de cuentas compartidas, sin dejar de lado la igualdad de género.

Esta visión de educación, emanada de Incheon, también se fortalece 
cuando se indica la necesidad de desechar todas las formas de exclusión y marginación al robustecer la promoción de la inclusión y la equidad por medio de la educación como la base de una agenda pedagógica transformadora. Todo ello da lugar a la promoción de oportunidades de aprendizaje de calidad para todos a lo largo de la vida, en todos los lugares y a todo nivel educativo.

De esta manera, de acuerdo con los aportes anteriormente indicados, queda establecido que la Educación en Derechos Humanos es una necesidad de las sociedades para enfrentar los cambios y buscar alternativas de solución a los problemas mundiales; pues lo que se busca es transformar la sociedad positivamente al interiorizar el respeto por la dignidad humana y valorar las costumbres, normas y estilo de vida de cada sujeto, para poder integrar proporcionalmente un conjunto social en el que se preserven los derechos humanos.

Igualmente, es una finalidad esencial de la educación, de manera que debe ser vista como un verdadero aprendizaje de la realidad social, que otorga herramientas sobre la mejor forma de participar en la sociedad y permite la adquisición de conocimientos básicos para la vida.

Rodino (2015) afirma que la Educación en Derechos Humanos "aspira a prevenir violaciones de derechos humanos, vista sustantivamente, busca producir las transformaciones individuales y sociales necesarias para crear comunidades sustentadas en una cultura de derechos humanos incluyentes, justas, equitativas $\mathrm{y}$ solidarias-" (p. 89).

En resumen, y siguiendo a Magendzo (2006), es posible definir la Educación en Derechos Humanos como la praxis educativa que se basa en el respeto, el reconocimiento, la defensa y la promoción de los derechos humanos, que busca el desarrollo de las personas como sujetos de derecho y que además tiene por objeto la aplicación de herramientas para que estos se hagan efectivos. Por lo tanto, sus fines deben encaminarse a la promoción de valores como el respeto a la dignidad de los individuos para la transformación de la sociedad.

La Educación en Derechos Humanos procura establecer una perspectiva de mundo basada en la cultura de los derechos de los individuos y de sus comunidades, de manera que estos se constituyan en ejes reguladores de las conexiones que se forman entre los pueblos. Debe ser vista como un verdadero aprendizaje de la realidad social, a tal grado de que influya esta visión en el modo de vida, las costumbres $\mathrm{y}$ los valores de un grupo.

Los docentes no solo deben enseñar contenidos, sino también actitudes, hábitos, costumbres y estrategias para el ejercicio y la defensa de las leyes, así como de la aceptación hacia los demás. Deben favorecer procesos de aprendizaje que permitan apropiarse de los conceptos sobre derechos humanos. Además, deben valorar todos los derechos humanos por igual (sociales, económicos, civiles, políticos). Todos poseen carácter enfático.

Lo anterior implica guiar al estudiantado en el manejo de situaciones difíciles para que se vaya dando un proceso en el que aprendan a ser responsables y autónomos, de manera que mejoren su compromiso cívico al sumarse a los demás en comunidad para resolver problemas y trabajar por un Estado justo, pacífico y democrático.

En correspondencia con esta tarea, el docente debe situarse en un nuevo paradigma, en que deje de ser mediador y se reconozca a sí mismo como un agente de cambio dentro de la sociedad y de la institución educativa. Además, debe incorporar el fomento del libre ejercicio de 
la curiosidad por medio de la organización del conocimiento. Así, evitará la acumulación estéril y garantizará el desarrollo de la capacidad de contextualizar los saberes en general y particularmente los que proporciona la Educación en Derechos Humanos, que están fundamentados en el ejercicio de la libertad y que. por tanto. anima a superar la discriminación y el respeto por la libertad de los demás.

\section{La propuesta político-pedagógica de Paulo Freire}

La pedagogía freireana está fundamentada en la idea de que la educación lleva a superar las prácticas discriminatorias e invisibilizadoras. De esta manera, conduce a la liberación del ser humano. Toda esta propuesta educativa se centra en la esperanza movilizadora y en el sueño de la utopía para un mundo mejor. "Un mundo donde mujeres y hombres se hallen en proceso de liberación permanente" (Freire, 2005, p. 62).

Según Freire (2005), los seres humanos construimos nuestra realidad a partir de nuestras vivencias como seres sociales, culturales, históricos y políticos. Nos convertimos en sujetos que efectúan su lectura crítica del mundo, con vistas a innovar y mejorar, en un diálogo permanente y una relación estrecha, pero diferente, con el conocimiento y la sociedad.

Sin embargo, la esperanza carece del poder de transformar la realidad por sí sola; es necesaria, pero no suficiente. Por esta razón, para lograr esa transformación, se debe unir la esperanza a la acción que cambiará la historia por venir. Se considera la vivencia sociocultural de todos los sujetos en cuanto necesidad ontológica la esperanza necesita de la práctica para volverse historia concreta. Por eso no hay esperanza en la pura espera, ni tampoco se alcanza lo que se espera en la espera pura, que así se vuelve espera vana (Freire, 2005, p.25).

Así, la esperanza se convertirá en un proyecto pedagógico que junto con el compromiso social serán recursos para transformar aquellas situaciones que se consideran injustas en la realidad.

Afirma Freire: “... la esperanza y su pedagogía siempre parten de una situación límite, y es donde surge la pedagogía de la esperanza y su materialización: lo inédito viable" (Freire, 2005 , p.125). Esta categoría implica que el sueño por un mundo mejor es posible y vendrá si las personas, que somos sujetos históricos, luchamos por conseguirlo. Es, entonces, en lo inédito viable donde se unen la acción y el pensamiento, haciendo posible la esperanza.

Para Freire (2005), en la esperanza se fundamentan la verdad y la calidad ética de la lucha. Ello permitió prescindir de la necesidad por el pasado. Así, resulta pertinente, en general, educar para superar la nostalgia y la visión de una realidad que no es tal y aceptarla como se presenta.

Ligada a la esperanza, aparece una pedagogía liberadora, que busca un diálogo permanente $\mathrm{y}$ una relación diferente con el conocimiento y con la sociedad. Además, transformará la política y la cultura. Aquí, la persona educadora debe promover una conciencia diferente sobre su labor y lucha por ayudar a develar la verdad al colaborar para que se descubran nuevas posibilidades y se superen los obstáculos que impiden encontrar la esperanza.

De acuerdo con esta nueva conciencia, las personas educadoras entienden que deben enseñar no solo 
contenidos, sino también la forma de abordarlos mediante una cultura del diálogo y una educación que suscite la reflexión.

E1 docente debe siempre considerar la realidad de los estudiantes y el hecho de que cada uno tiene tras de sí una experiencia personal, la cual le sirve para guiar sus actos y su comportamiento social y debe partir de esta lectura para enseñar contenidos (Freire, 2005). Además, debe contemplar que cada individuo concibe a la educación de forma distinta devenida de su entorno y experiencia de vida. Al comprender su realidad, se puede empezar a cambiar lo concreto y es posible porque se respeta la libertad y las características individuales de quienes aprenden.

Aunado a lo anterior, el reto del docente será pasar del discurso sobre su propia lectura del mundo a desafiar al alumnado para que hable sobre la suya. De esta forma, "para el educador o la educadora progresistas no hay otro camino que el de asumir el 'momento' del educando, partir de su'aquî y de su'ahora', para superar en términos críticos, con él, su ingenuidad" (Freire, 2005, p. 65) y esto será educar en, para y desde la libertad.

Al comprender así la acción educadora, el docente debe preguntarse acerca del potencial de cada uno de los educandos para actuar, reflexionar $\mathrm{y}$ disentir. Él o ella debe situarse "como sujeto que es capaz de conocer y que quiere conocer en relación con otro sujeto igualmente capaz de conocer, el educador, y entre los dos, posibilitando la tarea de ambos, el objeto del conocimiento" (Freire, 2005, p. 66).

De esta forma, el estudiante se relaciona con contenidos y descrubre qué puede conocer y así se inicia un proceso de aceptación de sí mismo como sujeto cognoscente con su propio sentido crítico. En congruencia, la práctica docente toma un nuevo sentido $y$ surgen las posibilidades para que el conocimiento lo produzca el mismo educando.

Aprender, con base en esta línea, significa para el estudiante la apropiación del contenido en estudio y el aprender a aprender, una manera progresista de ver su proceso de aprendizaje. En otras palabras, el estudiante será capaz de realizar un aprendizaje significativo de manera individual $\mathrm{y}$ en múltiples circunstancias, mientras desarrolla estrategias cognitivas que le permitan regular su propia actividad educativa y formarse para el ejercicio de la libertad.

Nos encontramos ante un enorme desafío que se le presenta al sistema educativo y es el de promover en los estudiantes, su actuación como individuos que experimenten el debate y que ejercitan el discernimiento para el análisis de los problemas que se le presentan a él y a la sociedad. (Freire, 2015, p. 87).

Lo anterior no significa que el respeto por el otro limite las posibilidades de aprender; todo lo contrario, se trata de visibilizar lo vivido y de tomarlo en cuenta, para que a partir de ahí construir el aprendizaje y darle un nuevo sentido, siempre al relacionarlo con la realidad de cada sujeto y su sapiencia. Esto garantizará una cultura de diálogo.

Esta disponibilidad para el diálogo es una postura que se debe asumir en el proceso de aprendizaje, donde "unos enseñan, y al hacerlo aprenden y otros aprenden, y al hacerlo enseñan" (Freire, 2005, p. 138). De igual forma, este "diálogo pedagógico implica el contenido $\mathrm{u}$ objeto cognoscible alrededor del cual gira [así] como la exposición hecha por el educador $\mathrm{o}$ la educadora para los educandos sobre ese contenido" (Freire, 2005, p.146).

Los docentes deben promover el diálogo constantemente para así lograr un aprendizaje crítico y creativo. Es mediante el diálogo que, según Freire, se produce la 
creación y la transformación del conocimiento y la construcción de la realidad social de cada uno de los participantes, lo que, a su vez, permite la transformación social. En otras palabras, mediante la interlocución, las personas aprenden y toman conciencia de que son sujetos de derecho y pueden reconstruir su realidad o mejorarla, de acuerdo con sus vivencias.

Así, existe interdependencia entre el acto de educar y el educador. El educador es educado también por el educando; es decir, mediante esta pedagogía dialógica, "el educador ya no es sólo el que educa sino aquel que, en tanto educa, es educado a través del diálogo con el educando, quien, al ser educado, también educa" (Freire, 1970, p. 61). Por esta razón, se habla de un proceso de enseñanza-aprendizaje, debido a que ambas partes enseñan y aprenden como conjunto, así que esta nueva forma de educación supera la oposición que se da entre educador y educandos, propia de la educación bancaria, que sirve a la dominación.

Lo anterior no significa el desdeño por la clase expositiva impartida por el o la docente. La pedagogía freiriana pretende erradicar la clase donde la persona educadora deposita o transfiere el conocimiento acumulado a sus estudiantes, donde no se les desafíe a pensar críticamente, a participar en el desarrollo de la clase y a profundizar en el tema que se está discutiendo.

Estas

proposiciones

politicopedagógicas de Paulo Freire reconocen que la educación no es neutra. Por un lado, en el proceso de enseñanza, se transmiten los propios pensamientos ideológicos; porel otro, siempre se deben respetar las ideas antagónicas de los alumnos. La educación, vista así, plantea el hecho de que educar no es solamente enseñar teoría, sino también llevarla a la reflexión. Ello permite tomar conciencia de quiénes somos y realizar acciones que nos permitan liberarnos de las situaciones de opresión para transformar el mundo que nos rodea y procurar una sociedad más justa y respetuosa de la dignidad de cada ser.

La educación tiene sentido porque las mujeres y los hombres aprendieron que se hacen $\mathrm{y}$ se rehacen aprendiendo, porque las mujeres y los hombres pudieron asumirse como seres capaces de saber, de saber que saben, de saber que no saben. De saber mejor lo que ya saben, de saber lo que todavía no saben. La educación tiene sentido porque, para ser, las mujeres y los hombres necesitan estar siendo. Si las mujeres y los hombres simplemente fueran, no habría por qué hablar de educación. (Freire, 2005, p. 50).

Siguiendo con la conciencia de mundo, Freire se expresa de esta forma:

La conciencia del mundo, que hace posible la conciencia de mí, hace imposible la inmutabilidad del mundo. La conciencia del mundo y la conciencia de mí me hacen un ser no sólo en el mundo sino con el mundo y con los otros. Un ser capaz de intervenir en el mundo y no sólo de adaptarse a él. En este sentido, las mujeres y los hombres interfieren en el mundo mientras que los otros animales sólo se mueven en él. Por eso, no sólo tenemos historia, sino que hacemos la historia que a su vez nos hace y que, en consecuencia, nos hace históricos. (Freire, 2005, p. 50).

Esta doble concepción pedagógica es fundamental para tomar conciencia de que en el proceso educativo la persona está llamada a convertirse en sujeto histórico y en creador de su propia realidad. 


\section{La Educación en Derechos} Humanos y la pedagogía de Freire

La pedagogía freireana está íntimamente relacionada con la Educación en Derechos Humanos; de hecho, de acuerdo con datos históricos, esta se inicia en Latinoamérica a partir de las luchas llevadas a cabo por los movimientos políticos en favor de las mujeres, los trabajadores, el ambiente, las minorías étnicas y sociales y la paz. Estas acciones incorporan el enfoque de Freire para lograr un cambio social y superar las estructuras sociales opresivas.

La Educación en Derechos Humanos busca que las personas sean capaces de distinguir la situación en que viven y participar así en la lucha por lograr nuevas posiciones históricas, políticas, sociales y culturales, por lo que es una educación política. A la vez, se alude a que "intenta formar personas comprometidas con la transformación de la sociedad en una más justa e igualitaria y en la cual los derechos humanos sean parte integral de la cultura ciudadana" (Magendzo, 2016, p.68).

Abogar por una educación con una función política es el primer aspecto básico de encuentro entre la Educación en Derechos Humanos y la pedagogía de Paulo Freire. Este pensador defiende que educar no es solo proporcionar conocimientos, sino también implica un uso real a la educación, de manera que esta se convierta en una herramienta para superar las injusticias sociales.

Según el autor, la educación deber ser vista como develadora de lo real y como la vía para reconocer el funcionamiento de la sociedad, su historia, el papel de los movimientos populares y otros hechos importantes para la construcción de una sociedad diferente e igualitaria. Además, proporciona elementos para encontrar la razón de ser de esos hechos sin ocultar o disfrazar la realidad.

Así que ambos enfoques colocan a las personas como sujetos en posición de transformar o crear -con ayuda de la educación- una sociedad diferente, donde se superen las estructuras sociales que subyugan e invisibilizan. Además, conciben la necesidad de analizar y visibilizar los comportamientos individuales y sociales que no están de acuerdo con la dignidad humana; igualmente, el darle el valor que requiere el hecho de investigar, difundir, planear y realizar acciones que generen cambios en las actitudes con el objeto de favorecer relaciones respetuosas de la igualdad entre todos los seres humanos.

Esta amalgama entre Freire y la Educación en Derechos Humanos crea, igualmente, una persona que se ve a sí misma como sujeto histórico, que puede elegir e intervenir en la realidad al transformarla, pero que es consciente de su inacabamiento (Freire, 2005), lo cual a su vez permite reconocerse como "seres inconclusos y siempre en desarrollo" (Rodino, 2015, p. 157).

De igual forma y conforme a lo anterior, un análisis permite reconocer como ambos enfoques pedagógicos se interesan en estudiar las estructuras de poder que se erigen en el sistema educativo. El enfoque freireano examina la forma en que "la estructura educativa y el currículo interactúan y dan forma al conocimiento. Mientras que, la Educación en Derechos Humanos se preocupa especialmente por cómo la estructura educativa y el currículo tienen efecto en moldear al sujeto de derechos" (Magendzo, 2003, p. 22).

Esta forma de educar exige un cambio en el sistema educativo tradicional, que sitúa al docente como el transmisor del conocimiento y que desconoce al estudiante como sujeto de derecho. En palabras de Freire, los 
docentes deben superar la educación bancaria como "una educación que, respetando la comprensión del mundo de los educandos, los desafíe a pensar críticamente, en primer lugar, y que no separe la enseñanza del contenido del enseñar a pensar correctamente" (Freire, 2005, p.202).

Para ello, deben utilizar métodos que encaminen a los estudiantes a obtener control sobre sus aprendizajes y se convierten en personas que aprendan de forma independiente $\mathrm{y}$ con mayor disposición para desarrollar posiciones críticas. En otras palabras, los enfoques pedagógicos mencionados deben tomar una posición de cuestionamiento contra la estructura tradicional del sistema educativo y contra las formas en que se realiza el aprendizaje. Así, se contribuye a un cambio.

La pedagogía freireana, de acuerdo con Magendzo (2003), está diseñada "con el propósito de capacitar a quien aprende para que se vuelva consciente de los condicionamientos en su vida y en la sociedad y disponga de las habilidades, conocimiento y recursos para poder planificar y crear cambios" (p. 24). En esta misma línea, la Educación en Derechos Humanos considera que el aprendizaje debe ayudar a vincular a las personas con la búsqueda de soluciones a los grandes problemas sociales tales como la pobreza, la injusticia, la discriminación, la intolerancia, etc.

La Educación en Derechos Humanos debe desarrollarse en un ambiente educativo libre de restricciones o de imposiciones rígidas con promoción del diálogo y la comunicación. Esta atmósfera debe basarse en la libre expresión y en un plano de igualdad y respeto entre educandos y el educador, en el que unos y otros se expresen y se escuchen. El acto educativo nunca puede ser jerarquizado, donde el instructor está por encima del estudiante, porque en relación con los derechos humanos "nadie lo sabe todo, ni nadie lo ignora todo. Todas las personas de cualquier edad o condición poseemos conocimiento $\mathrm{o}$ experiencias vitales que al compartirse enseñan algo a los demás y los enriquece" (Rodino, 2015, p. 197).

En la Educación en Derechos Humanos, el diálogo se entiende "como proceso epistemológico, es decir, como vía para aprender sobre cierto objeto de conocimiento en cuya búsqueda educadores y educandos se comprometen juntos" (Rodino, 2015, p. 208). Esta visión dialógica de la educación es la misma de la que habla Freire, la opuesta a la educación bancaria, y que considera al diálogo como aquella "posibilidad de que dispongo para, abrirme al pensar de los otros y no perecer en el aislamiento" (Freire, 2005, p. 148).

Todo lo expuesto anteriormente trae implicaciones para el quehacer del docente, porque ahora debe utilizar estrategias que promuevan un clima en el aula de igualdad y reconocimiento por los demás y donde también la persona docente implemente la práctica de la coherencia que implica reducir la distancia entre lo que decimos y lo que hacemos de manera que se logre "disminuir la distancia entre el discurso y la práctica." (Fallas, 2012, p. 192).

De la misma forma, sobre el hecho educativo, Freire indica que ahora se dará una interdependencia diferente entre el acto de enseñar y el acto de aprenderAhora, el educador también será instruido por el educando, de manera que esta nueva forma de educación supera la tradicional contradicción entre educador y educandos, propia de la formación bancaria, que sirve a la dominación, "... ahora, ya nadie educa a nadie, así como tampoco nadie se educa a sí mismo, los hombres se educan en comunión, y el mundo es el mediador" (Freire, 1970, p. 61). 
Igualmente, se debe conocer la realidad sin ocultar el conflicto y la polémica y ayudar a los estudiantes "a la indagación crítica y a la búsqueda creadora" (Rodino, 2015, p. 212). Para educar, los docentes deben desarrollar la capacidad de practicar una pedagogía de la pregunta como la que propone Freire, la cual estimula a los niños y les desarrolla "el gusto por la pregunta, la pasión del saber y de la curiosidad, la alegría de crear y el placer del riesgo, sin el cual no hay creación" (Freire, 2005, p. 171).

Por medio de la pregunta, el estudiante ejercita la reflexión y construye su propio conocimiento. El preguntar le abre la posibilidad de conocer los problemas de la sociedad, lo cual crea la oportunidad para desarrollar competencias afectivas, sociales y cognitivas para ser un ciudadano crítico, defensor de los derechos humanos y capaz de realizar un cambio social.

\section{Consideraciones finales}

La Educación en Derechos Humanos se centra en la construcción de un sujeto de derecho, consciente de los condicionamientos en su vida, en la sociedad y comprometido con la promoción, así como en la realización de cambios sociales que incidan en el modo de vida, las costumbres y los valores para instituir una cultura de respeto $y$ valoración de la dignidad de la persona, donde se practiquen la justicia social, la inclusión, la equidad y la solidaridad.

La propuesta pedagógica de Paulo Freire parte del reconocimiento del educando como sujeto cognoscente $\mathrm{y}$ como creador de su propia historia, a la vez, es reflexivo y cuestionador de su realidad, interlocutor en la construcción de un cambio social que incida en las condiciones materiales de la sociedad. Esta educación de la mano con una cultura de diálogo debe servir a tomar conciencia y ser liberadora.

La Educación en Derechos Humanos y la pedagogía freireana concuerdan en que la educación tiene una función política ineludible de transformación de la sociedad. De igual forma, ambos enfoques defienden la necesidad de tomar distancia de la educación tradicional y las estructuras de poder dentro del sistema educativo $\mathrm{y}$ dentro del aula. De esta manera, el hecho educativo se basará en diálogo, acortará las distancias creadas por las jerarquías, modificará la realidad educativa y preparará el compromiso social.

Queda demostrado por lo que se ha argumentado que la Educación en Derechos Humanos y la pedagogía propuesta por Paulo Freire coinciden en su esencia y en sus propósitos de lograr un cambio educativo que promueva una cultura de paz, justicia social y democracia al visibilizar las situaciones que arriesgan la dignidad de los seres humanos.

\section{Referencias bibliográficas}

Achkar, S. (2002). Una mirada a la educación en derechos humanos desde el pensamiento de Paulo Freire. Prácticas de intervención politico-cultural. Recuperado de: http://www.corteidh.or.cr/tablas $/$ r24462.pdf

Fallas, C. (2012). Educación para la paz: fomentando valores contra todo tipo de violencia. San José: EUNED.

Freire, P. (1970). Pedagogía del oprimido. México: Siglo XXI Editores.

Freire, P. (1996). Pedagogia da autonomia: saberes necessários à prática. Paz e Terra, Coleção Leitura. São Paulo. Recuperado de: http://forumeja.org.br/files/Aut onomia.pdf 
Freire, P. (2005). Pedagogía de la esperanza: un reencuentro con la pedagogía del oprimido. México: Siglo XXI Editores. Recuperado de: http://www.cronicon.net/pagina s/Documentos/paq2/No.11.pdf

Freire, P. (2015). Educación como práctica de la libertad. México: Siglo XXI Editores.

Instituto Interamericano de Derechos Humanos. (2002). Desarrollo normativo. En Informe interamericano de la educación en derechos humanos. Un estudio en 19 países (parte I). San José: Editorama, S.A. Recuperado de: http://www.iidh.ed.cr/multic/U serFiles/Biblioteca/IIDH/3_2010 /a8618e63-68ec-4d5d-90e1$5 f f 4 f 479 d c a 4 . p d f$

Korol, C. (1993). Como nunca en la historia, Marx pagaría por vivir en el mundo de hoy. Entrevista al pedagogo brasileño Paulo Freire. Recuperado de: http://www.portalces.org/bibliot eca/equidad-social/entrevista-alpedagogo-brasileno-paulo-freirecomo-nunca-historia-marx

Magendzo, A. (1996). Currículum y educación para la democracia en la modernidad. Instituto para el Desarrollo de la Democracia "Luis Carlos Galán", Bogotá: Antropos.

Magendzo, A. (2003). Pedagogía crítica y educación en derechos humanos: Paulo Freire. Revista de Pedagogía Crítica, 2, 19-27. Recuperado de: https://doi.org/10.25074/071955 32.2.516

Magendzo, A. (2004). Formación ciudadana. Bogotá: Cooperativa Editorial Magisterio.

Magendzo, A. (2006). Educación en Derechos Humanos: un desafio para los docentes de hoy. Santiago: LOM Ediciones.

Magendzo, A. (2007). El derecho a la educación: una reflexión desde el paradigma crítico y la Educación en Derechos Humanos. En Módulos Sistematizados en Derechos Humanos. Programa de Derechos Humanos, Universidad Nacional, Heredia, Costa Rica.

Magendzo, A. (2012). Algunos principios pedagógicos orientadores de la Educación en Derechos Humanos. Revista Decisiones, 32, Centro de Cooperación Regional para la Educación de Adultos en América Latina y el Caribe, Ciudad de México, México.

Magendzo, A. (2015). Educación en Derechos Humanos y Educación Superior: una perspectiva controversial. Reencuentro, núm. 70, pp. 47-69. Recuperado de: http://www.redalyc.org/articulo. oa?id=34046812004

Magendzo, A. (2016). Incorporando la perspectiva controversial en el currículum disciplinario. Revista Iberoamericana de Educación Superior, 7, núm. 19, 118-130. Recuperado de: http://www.redalyc.org/pdf/299 1/299145847007.pdf

Morin, E. (2008). Reformar la educación, la enseñanza, el pensamiento. Recuperado de: https://bcehricardogaribay.word press.com/2009/08/16/reformarla-educacion-la-ensenanza-elpensamiento/

Organización de las Naciones Unidas, Asamblea General (1948). Declaración Universal de Derechos Humanos. París, Francia. Recuperado de: https://www.un.org/es/universal -declaration-human-rights/ 
Organización de las Naciones Unidas, Asamblea General (1989). Convención sobre los Derechos del Niño. Nueva York, Estados Unidos. Recuperado de: https://www.ohchr.org/sp/profe ssionalinterest/pages/crc.aspx

Organización de las Naciones Unidas, Asamblea General (1999). Observación General n. ${ }^{\circ}$ 13: El derecho a la educación. Comité de Derechos Económicos, Sociales y Culturales, período 21 de sesiones. Nueva York, Estados Unidos. Recuperado de: https://confdts1.unog.ch/1\%20SPA/Tradute k/Derechos_hum_Base/CESCR/ 00_1_obs_grales_Cte\%20Dchos\% 20Ec\%20Soc\%20Cult.html\#GEN 13

Organización de las Naciones Unidas, Asamblea General (2004). Programa Mundial de las Naciones Unidas para la Educación en Derechos Humanos (2005-2007). Recuperado de: https://documents-ddsny.un.org/doc/UNDOC/GEN/ N04/483/07/PDF/N0448307.pd f?OpenElement

Organización de las Naciones Unidas. (2006). Plan de acción. En Programa Mundial para la Educación en Derechos Humanos (primera etapa). París: UNESCO. Recuperado de: http://www.ohchr.org/Documen ts/Publications/PActionEducatio nsp.pdf

Organización de las Naciones Unidas. (2011). Declaración de las Naciones Unidas sobre Educación y Formación en Materia de Derechos Humanos. New York, Estados Unidos. Recuperado de: http://www.derechoshumanos.u nlp.edu.ar/assets/files/document os/declaracion-de-nacionesunidas-sobre-educacion-y- formacion-en-materia-dederechos-humanos.pdf

Organización de Naciones Unidas, Asamblea General (2011) Declaración sobre la Educación y Formación en Materia de Derechos Humanos. Resolución de la Asamblea General A/C.3/66/I.65, del 19 de diciembre de 2011. Recuperado de:

http://www.derechoshumanos.u nlp.edu.ar/assets/files/document os/declaracion-de-nacionesunidas-sobre-educacion-yformacion-en-materia-dederechos-humanos.pdf

Organización de las Naciones Unidas (2018). Resolución 39/3. Programa Mundial de las Naciones Unidas para la Educación en Derechos Humanos (cuarta fase). Ginebra, Suiza. Recuperado de: https://documents-ddsny.un.org/doc/UNDOC/GEN/ G18/293/78/PDF/G1829378.pd f?OpenElement

Rodino, A. (2015). Educación en derechos humanos para una ciudadanía democrática. San José: EUNED.

Tancara, C. (1993). La investigación documental. Temas Sociales, 17, 91106. La Paz. Recuperado de http://www.scielo.org.bo/scielo. php?pid=S0040$29151993000100008 \&$ script $=$ sci arttext

UNESCO (1990). Declaración Mundial de Educación para Todos. Jomtien, Tailandia. Recuperado de

https://www.oei.es/historico/efa 2000jomtien.htm

UNESCO (2015). Declaración de Incheon: "Educación 2030: Hacia una Educación Inclusiva y Equitativa de Calidad y un Aprendizaje a lo Largo de la Vida 
Puntos de encuentro entre el enfoque pedagógico de Paulo Freire y la Educación en Derechos Humanos

para Todos". Foro Mundial sobre la

Educación, Incheon, Korea.

Recuperado de

https://unesdoc.unesco.org/ark:/

48223/pf0000233137_spa

Recibido: 05 de noviembre de 2019

Aceptado: 20 de mayo de 2020 